\title{
Preliminary Study on Phenotypic Detection of ESBL and Amp-C $\beta$-Lactamase-Producing Escherichia Coli Isolates from Slaughtered Pigs
}

\author{
Marinela TIPIȘC $\breve{A}^{1}$, Andreea Paula COZMA ${ }^{2 *}$, Dragoș ANIȚĂ ${ }^{2}$, Elena IȘAN ${ }^{1}$, Luanda \\ OȘLOBANU ${ }^{2}$, Adriana ANIȚA ${ }^{2}$, Gheorghe SAVUȚA ${ }^{2}$
}

\author{
${ }^{1}$ Sanitary-Veterinary and Food Safety Laboratory, (Iasi, 700489, Romania) \\ ${ }^{2}$ University of Agricultural Sciences and Veterinary Medicine, (Iasi, 700490, Romania) \\ * Corresponding author: A. P. Cozma e-mail: andreeapaulacozma@yahoo.com
}

\section{SHORT COMMUNICATION}

\begin{abstract}
The presence of extended-spectrum beta-lactamase-producing Escherichia coli in food animals is a public health concern. The aim of this study was to phenotypically detect the extended $\beta$-lactamase and AmpC $\beta$-lactamase-producing E. coli isolated from pig caecum from three slaughterhouses in the North-East of Romania. After collecting the cecal samples, they were phenotypically processed. ESBL and AmpC screening was carried out by cultivation on MacConkey medium with addition of cefotaxime (MC+CTX). The isolates were confirmed as being E. coli based on the biochemical properties using MIU, TSI and API 20E and as ESBL based on synergy tests between clavulanic acid and ceftazidime and/or clavulanic acid and cefotaxime. Phenotypical detection of AmpC beta-lactamase-producing strains was carried out through a stable AmpC cephalosporin (cefepime). Following ESBL/AmpC screening, out of the 128 analysed samples, 51 (39.84\%) grew on MC+CTX and they were identified as E. coli. After performing microdilution in broth using EUVSEC2 plates, 78.43\% isolates were identified with ESBL phenotype, 9.8\% isolates with ESBL/AmpC phenotype, and $11.77 \%$ isolates with AmpC phenotype. In this study, although a limited number of strains were analysed, the obtained results phenotypically confirm the presence of ESBL and AmpC enzymes in slaughtered pig populations from the North-East of Romania.
\end{abstract}

Keywords: AmpC; E. coli; ESBL; slaughtered pigs.

Received: 27 January 2021

Accepted: 22 February 2021

Published: 14 May 2021

DOI:

10.15835/buasvmcn-vm:2021.0001

(1) $\Theta \Theta \Theta(2021$ Authors. The papers published in this journal are licensed under the Creative Commons Attribution-NonCommercialNoDerivatives 4.0 International License

\section{INTRODUCTION}

Extended-spectrum beta-lactamase (ESBL) enzyme and AmpC beta-lactamase enzyme-producing Enterobacteriaceae strains represent a major public health concern as a result of the resistance to third-generation cephalosporins, antibiotics that are commonly used both in human and in veterinary medicine (Salviati et al., 2014). The carriage of ESBL and AmpC enzymes and the risk of their transmission from production animals to humans through various routes (direct contact, contaminated food) is known and signalled in the scientific literature (Kola et al., 2012; Dahms et al., 2015). Several studies from Europe revealed the association of consumption of cephalosporins in pig farms with the high prevalence of ESBL-producing E. coli isolates (Hammerum et al., 2014; Dahms et al., 2015; Schmithausen et al., 2015).

The aim of the study was to assess the current situation regarding the faecal carriage with positive ESBL/AmpC E. coli strains isolated from the caecal content of slaughtered pigs. 


\section{MATERIALS AND METHODS}

Isolation of ESBL/AmpC-producing Escherichia coli strains from the samples of swine caecal content was carried out according to the European Reference Laboratory for Antimicrobial Resistance (EURL-AR) (Hasman et al., 2018). After sterile collection of caecal content, a pre-enrichment was carried out first using peptone water. The ESBL/AmpC screening was performed using the selective MacConkey medium (Oxoid, Basingstoke, UK), with the addition of cefotaxime. All colonies with morphology typical for Escherichia coli were confirmed based on the biochemical properties using the triplesugar-iron (TSI) agar test, the motility-indole-urea (MIU) medium or Api 20E for atypical E. coli strains. For phenotypical confirmation of ESBL isolates, the microdilution method was carried out in broth using EUVSEC2 plates that contain cefoxitine, cefepime, cefotaxime, ceftazidime and clavulanate combined with cefotaxime and ceftazidime. The results were interpreted based on the synergy tests between clavulanic acid and ceftazidime and/or clavulanic acid and cefotaxime. Phenotypical detection of AmpC beta-lactamase-producing strains was carried out through a stable AmpC cephalosporin: cefepime.

\section{RESULTS AND DISCUSSIONS}

A total of 128 samples of caecal content were analysed of which, following ESBL/AmpC screening, 51 (39.84\%) presumptive extended-spectrum beta-lactamase enzyme-producing and/or AmpC enzyme-producing E. coli strains have been isolated and identified. The synergy test between the potentiated cephalosporin and the simple cephalosporin phenotypically confirmed that $100 \%$ of the $E$. coli isolates identified following screening are presumptive ESBL and/or AmpC-producing isolates. Also, following centralization of the results obtained from the 51 E. coli isolates of animal origin, attained following ESBL/AmpC screening, most of them showed ESBL phenotype (40/51; 78.43\%), 5/51 (9.80\%) showed ESBL+AmpC phenotype, and 6/51 (11.77\%) showed AmpC phenotype (Table 1).

Table 1. Phenotypes identified in E. coli isolates

\begin{tabular}{|c|c|c|c|c|}
\hline \multirow[b]{2}{*}{$\begin{array}{c}\text { Source of } E \text {. coli } \\
\text { strains isolated on } \\
\text { MC+CTX }^{\dagger}\end{array}$} & \multirow[b]{2}{*}{$\begin{array}{l}\text { No. of strains } \\
\text { isolated on } \\
\text { MC+CTX* }\end{array}$} & \multicolumn{3}{|c|}{ Type of phenotype } \\
\hline & & $\begin{array}{c}\text { No. of } E \text {. coli strains } \\
\text { phenotype ESBL } \\
(2016+2017)\end{array}$ & $\begin{array}{c}\text { No. of } E \text {. coli strains } \\
\text { phenotype } \\
\text { ESBL+AmpC } \\
(2016+2017)\end{array}$ & $\begin{array}{c}\text { No. of } E \text {. coli strains } \\
\text { phenotype AmpC } \\
(2016+2017)\end{array}$ \\
\hline $\mathrm{IS}^{\dagger}$ slaughterhouse & 6 & 5 & 1 & 0 \\
\hline $\mathrm{BT}^{\dagger}$ slaughterhouse & 32 & 26 & 3 & 3 \\
\hline $\mathrm{SV}^{\dagger}$ slaughterhouse & 13 & 9 & 1 & 3 \\
\hline $\begin{array}{l}\text { Total isolates of } \\
\text { animal origin }\end{array}$ & 51 & 40 & 5 & 6 \\
\hline
\end{tabular}

$\mathrm{MC}+\mathrm{CTX}^{\dagger}$ - MacConkey medium with the addition of cefotaxime; $\mathrm{IS}^{\dagger}$ - Iași; $\mathrm{BT}^{\dagger}$ - Botoșani; $\mathrm{SV}^{\dagger}$ - Suceava

For swine, the faecal carriage with ESBL enzyme-producing E. coli strains has big geographic differences, with proportions that vary between $15.2 \%$ in Switzerland (faecal samples) and $23.4 \%$ in the United Kingdom (caecal samples) or 49\% in Portugal (faecal samples) (Geser et al., 2011; Ramos et al., 2013; Randall et al., 2014). Furthermore, in Belgium, the study carried out by Van Damme et al. (Van Damme et al., 2017) highlighted the presence of positive ESBL/AmpC E. coli strains in $75 \%$ of the samples of faecal batches and $47 \%$ of the samples of tonsils collected upon slaughtering of pigs. The preliminary results from this study fall into the range listed in other similar studies carried out in Europe. Moreover, the big differences between various areas of Europe can be justified by the policy of each country on the administration of antibiotics in production animals. In some countries, such as the Netherlands, following a high prevalence obtained, starting January 2013, veterinarians have been legally forced to limit the use of third/fourth-generation cephalosporins and fluoroquinolones only in infections confirmed by bacteriological examinations and susceptibility tests (SDa, 2013).

Considering that pigs and, subsequently, pork add to the zoonotic spread of ESBL/AmpC E. coli strains (Carmo et al., 2014; Evers et al., 2017; Lazarus et al., 2015) and that ESBL/AmpC strains can persist in the human gastrointestinal track for up to 5 years (Brolund, 2014), intervention strategies are necessary to reduce the presence of these isolates on pig carcasses and to reduce the exposure of humans to ESBL/AmpC-producing enterobacteria.

\section{CONCLUSIONS}

In conclusion, the high percentage $(39.84 \%)$ of presumptive extended-spectrum beta-lactamase enzyme-producing and/or AmpC enzyme-producing E. coli strains isolated from the caecum of slaughtered pigs can represent an important source of contamination of carcasses and pork, respectively, and there is a risk of their transmission from production animals to humans through various routes.

Author Contributions: M.T. - collected the samples and performed the phenotypic analysis of clinical isolates; A.P.C. analyzed the data and wrote the manuscript; D.A. E.I, L.O., A.A - analyzed the data; G.S - planned and coordinated the study, analyzed the data and wrote the manuscript. 


\section{Acknowledgments}

This research did not receive any specific grant from funding agencies in the public, commercial, or not-for-profit sectors.

\section{Conflicts of Interest}

The authors declare that they do not have any conflict of interest.

\section{REFERENCES}

1. Brolund A. Overview of ESBL-producing Enterobacteriaceae from a Nordic perspective, Infect. Ecol. Epidemiol. 2014; 4, art. 24555.

2. Salviati Von C, Friese A, Roschanski N, Laube H, Guerra B, Kasbohrer A, et al. Extended-spectrum beta-lactamases (ESBL)/AmpC beta-lactamases-producing Escherichia coli in German fattening pig farms: a longitudinal study. Berl. Munch. Tierarztl. Wochenschr., 2014;127: 412-419.

3. Carmo LP, Nielsen LR, da Costa PM, Alban L. Exposure assessment of extended-spectrum beta-lactamases/AmpC beta-lactamases-producing Escherichia coli in meat in Denmark, Infect. Ecol. Epidemiol. 2014; 4, art. 22924.

4. Dahms C, Hübner NO, Kossow A, Mellmann A, Dittmann K, Kramer A. Occurrence of ESBL-producing Escherichia coli in livestock and farm workers in Mecklenburg-Western Pomerania, Germany. PLoS One, 2015; 10 . p. e0143326.

5. Evers EG, Vennemann FB, Wijnands LM, Chardo JE. Comparative exposure assessment of ESBL-producing Escherichia coli through meat consumption PLoS One, 2017; 12, Article e0169589.

6. Geser N, Stephan R, Kuhnert P, Zbinden R, Kaeppeli U, Cernela N et al. Fecal carriage of extended-spectrum betalactamase-producing Enterobacteriaceae in swine and cattle at slaughter in Switzerland. J. Food Prot., 2011; 74: 446449.

7. Hammerum AM, Larsen J, Andersen VD, Lester CH, Skytte TS, Hansen F et al. Characterization of extended-spectrum $\beta$-lactamase (ESBL)-producing Escherichia coli obtained from Danish pigs, pig farmers and their families from farms with high or no consumption of third- or fourth-generation cephalosporins. J Antimicrob Chemother., 2014; 69: 265057.

8. Hasman H, Agers $\varnothing$ Y, Hendriksen R, Cavaco LM, Guerra-Roman B. Laboratory protocol- Isolation of ESBL-, AmpCand carbapenem. European Union Reference Laboratory for Antimicrobial Resistance (EURL-AR); 2018; Available from: https://www.eurl-ar.eu/CustomerData/Files/Folders/21-protocols/530_esbl-ampc-cpeprotocol-version-caecalv7-09-12-19.pdf

9. Kola A, Kohler C, Pfeifer Y, Schwab F, Kühn K, Schulz K et al. High prevalence of extended-spectrum-beta-lactamaseproducing Enterobacteriaceae in organic and conventional retail chicken meat, Germany. J Antimicrob Chemother., 2012; 67: 2631-34.

10. Lazarus B, Paterson DL, Mollinger JL, B.A. Rogers. Do human extraintestinal Escherichia coli infections resistant to expanded-spectrum cephalosporins originate from food-producing animals? A systematic review Clin. Infect. Dis., 2015; 60:439-452.

11. Ramos S, Silva N, Dias D, Sousa M, Capelo-Martinez JL, Brito F, et al. Clonal diversity of ESBL-producing Escherichia coli in pigs at slaughter level in Portugal. Foodborne Pathog. Dis., 2013; 10: 74-79.

12. Randall LP, Lemma F, Rogers JP, Cheney TEA, Powell LF, Teale CJ. Prevalence of extended-spectrum-betalactamase-producing Escherichia coli from pigs at slaughter in the UK in 2013. J. Antimicrob. Chemother. 2014; 69:2947-2950.

13. Schmithausen RM, Schulze-Geisthoevel SV, Stemmer F, El-Jade M, Reif M, Hack S, et al. Analysis of transmission of MRSA and ESBL-E among pigs and farm personnel. PLoS One. 2015; 10: e0138173. pmid:26422606.

14. SDa (Netherlands Veterinary Medicines Institute). Usage of antibiotics in livestock in The Netherlands in 2012. Netherlands Veterinary Medicines Institute. 2013; Available https://www.autoriteitdiergeneesmiddelen.nl/en/news/16/sda-report-usage-of-antibiotics-in-livestock-in-thenetherlands-in-2013-now-available-in-english

15. Van Damme I, Garcia-Graells C, Biasino W, Gowda T, Botteldoorn N, De Zutter L. High abundance and diversity of extended-spectrum beta-lactamase (ESBL)-producing Escherichia coli in faeces and tonsils of pigs at slaughter. Vet. Microbiol. 2017; 208: 190-194. 